\title{
Modeling HIV Spread Through Sexual Contact Using a Cellular Automaton
}

\author{
Azadeh Alimadad*, Vahid Dabbaghian*, Suraj K. Singhk*, and Herbert H. Tsang*† \\ * Modelling of Complex Social Systems Program \\ Interdisciplinary Research in the Mathematical and Computational Sciences (IRMACS) Centre \\ Simon Fraser University, Burnaby, British Columbia, Canada \\ †Department of Computer Science \\ University of Calgary, Calgary, Alberta, Canada \\ Email: \{htsang\}@ucalgary.ca
}

\begin{abstract}
Having a risky sexual behavior increases the likelihood of infection by the Human Immunodeficiency Virus (HIV), which causes the Acquired Immunodeficiency Syndrome (AIDS). This has been a long lasting problem in high-risk populations such as sex workers: individuals in this population may face drug addiction and share infected needles, or have unprotected sex, and both issues can result in an HIV infection that may then be transmitted to other parts of the population. To study the dynamics of the HIV epidemic in such a high-risk community, we propose a model in which the population is represented as a cellular automaton. At the macro-level, our model accounts for the fact that the sexual behavior of an individual is influenced by the social norms of his acquaintances (social network) as well as by his awareness of HIV status. At the micro-level, randomized neighborhoods provide an explicit representation of personal interactions standing for the large number of nonrepeated encounters in populations at risk. Our simulations study the dynamics of the disease for different social norms as well as the probability that a seropositive individual get tested.
\end{abstract}

\section{INTRODUCTION}

In 2008, 2.7 million people became infected with HIV and 2 million deaths were ascribable to the infection, for a total of 33.4 million people living with HIV [1]. Thus, the HIV pandemic remains a major preoccupation for public health and a vast body of research continues to be devoted to the understanding of the transmission mechanisms and their associated risk factors. This understanding can be obtained by ways such as field work summarizing statistical data in specific populations, or by theoretical models bolstered by real-world data. Models can be used for recommendations (e.g., how to allocate resources in a given population), projections, or to analyze the interplay between different factors. Our approach focuses on the latter.

Research has shown that the social network of an individual (i.e., the characteristics of his acquaintances) plays a major role in his sexual behavior. Indeed, the community dictates behavioral norms to which individuals conform even if they are aware of the inherent risks, and this has prompted prevention strategies aiming at shifting norms in high-risk groups towards safer behaviors [2]. The role of acquaintances and the norms they convey is illustrated by the following two findings. Firstly, a homeless young adult, who belongs to a population at risk, can be coerced to trade sex by acquaintances, which increases the likelihood of risky behaviors [3]. Secondly, also for homeless young adults, the "odds of HIV-risk were lower with a greater number of peers who attend school, have a job, or have positive family relationships" [4]: in a nutshell, the more pro-social peers and the less HIV sex risk behavior [5]. Thus, the behavioral norms can have a negative (social pressure) or a positive (social support) impact, not only on the likelihood of the infection but also on the survival once infected [6]. Our model takes into account this important feature by considering that one's behavior depends on the balance of positive and negative influences received from acquaintances. We consider environmental influences to be expressed via individuals.

However, the social network exerts an influence on the individual but would fail to alone describe one's behavior. For example, an individual who is aware of his seropositivity may choose to disclose his status to his sexual partner, which may lead to less risky sexual activities [7] or to risky activities practiced only with other seropositive individuals [8]. On the other hand, an individual who is not aware of his seropositivity and yet receives the same social influences is likely to yield more acute risks. Thus, our model represents different states of the population using a compartmental framework [9], [10]. Concretely, we divide the population into epidemiological classes based on HIV status, awareness of seropositivity, and sexual behavior [11].

A crucial feature of compartmental frameworks is that they are homogeneous-mixing models. Concretely, they assume that all individuals have the same likelihood of infection. From a social network perspective, this is assuming that all individuals are in contact with each others, which is clearly not in agreement with the reality of HIV in humans: individuals have different social networks and this heterogeneity impacts the dynamics of infection [12]. Thus, we include heterogeneity in social networks using a cellular automaton (CA) [13]. This technique had been advocated to model complex social processes and diseases [14], [15], [16], and has seen a growing number of applications to the dynamics of HIV in the last decade [17], [18]. A side effect of using a CA is that we employ a discrete model, whereas a purely compartmental framework is a continuous model (it is formalized by differential equations which express the mean-field-like 
approximation of the corresponding CA model). Having a discrete model differentiates our approach from, for example, a continuous model on a square lattice with sexual contacts between neighbors [19]. Furthermore, we differ from such models by using randomized neighbors in the automaton to account for the large number of non-repeated encounters in high-risk populations [20] targeted by our approach, such as men who have sex with men and sex workers.

In Section II, we introduce the epidemiological classes of our model and how individuals flow between classes under the social influences of their acquaintances. In Section III, we study the dynamics resulting from improvements on positive influences and/or the probability that seropositive individuals get tested, and we summarize the overall behavior of the model. Finally, we discuss the main assumptions and we suggest improvements.

\section{THEORETICAL FRAMEWORK}

\section{A. Compartmental Model}

We consider a constant population divided into five epidemiological classes, depending on HIV status, awareness of seropositivity (i.e., infection by HIV), and sexual behavior. We use the abbreviation $\mathbf{H}$ to denote a class with a high-risk sexual behavior, and similarly $\mathbf{L}$ for a class with a low-risk sexual behavior. A class labelled with a ${ }^{-}$denotes non-seropositive individuals, while a ${ }^{+}$denotes seropositive individuals. In the latter, individuals may be unaware of their status, which we denote by a $\mathbf{U}$. One special class $\mathbf{D}$ accounts for seropositive individuals who decreased their high-risk sexual activities given their status. These combination yield the classes $\mathbf{L U}^{+}$, $\mathbf{H U}^{+}, \mathbf{H}^{-}, \mathbf{L}^{-}$and $\mathbf{D}$.

The features that we take into account to establish classes have been used in several other models. For example, the model in [21] had three classes based on seropositivity and awareness (thus roughly equivalent to $\mathbf{H}^{-} / \mathbf{L}^{-}, \mathbf{L U}^{+} / \mathbf{H U}^{+}$, and D) and a fourth one representing individuals with AIDS. Furthermore, heterogeneity in sexual behavior was considered by the model in [22] using changing partners. Among other important features found in models of HIV, delays can express that the likelihood of an individual to transmit the disease depends on his stage. We model delays by equipping individuals with counters, as detailed in the next section.

\section{B. Cellular Automaton}

We explicitly model an individual and his sexual partners at a given time step by using a cellular automaton. A cellular automaton is a regular grid of cells, each representing an individual. In this study, we use a common type of toroidal grid in two dimensions consisting of square cells. The state of a cell, or the epidemiological class of the associated individual, varies according to rules. The rules describe how individuals change simultaneously at each time step, as a result of who they interact with. In other words, they govern changes in individuals given social influences. Social influences can be broadly classified in two types: they can be safe such as being influenced by an individual in class $\mathbf{D}$ who opted for sexual practices with very low risks, or they can be unsafe when an individual with a high-risk sexual behavior influences an individual with a low-risk sexual behavior. The probability of being encouraged toward safe practices is denoted by $\alpha$, and the probability of encouragement toward unsafe influences is denoted by $\beta$.

In a high-risk population, there is a large number of nonrepeated encounters. Thus, we change the sexual partners at each time step by using a randomized neighborhood. Concretely, the partners of a given cell are always chosen among the cells in the immediate vicinity, but the content of the cells are randomly swapped. Another behavioral property drawn straightforwardly from the real world is that the more people are exerting an influence on an individual, and the more likely the individual changes his behavior. This is modelled by using a social counter for each cell. At each time step, the counter records the cumulative influences $\alpha$ and $\beta$ from sexual partners, for having safe or unsafe behavior respectively. The influence $\alpha$ is added whereas $\beta$ is subtracted, in order to model a balance. Formally, we assign to each epidemiological class a number, as shown in Figure 1. For an individual $i$, we denote by $R_{i, j, t}$ the number of his sexual partners in epidemiological class $j$ at time $t$. The influence exerted on $i$ by an individual in class $j$ is denoted $v_{j i}$. This influence can be seen as the probability that $i$ changes his behavior after a sexual relationship with an individual in $j$ in one unit of time. We abstract it as being either safe or unsafe, and all values are given in Figure 1; individuals in state $\mathbf{D}$ do not receive social influences, since it is considered that they do not change anymore. The social counter for $i$ has recorded an influence $C_{i}(t)$ at time $t$ expressed by:

$$
C_{i}(t)=C_{i}(t-1)+\sum_{j=0}^{4} v_{j i} R_{j}
$$

We also use two probabilities. The first one represents the probability $q$ for seropositive individuals who are unaware of their status to get tested. Being able to conduct simulations for different probabilities of testing and social influences is of particular interest, since studies have expressed a desire for more prevention than testing, due to considerations such as costeffectiveness. Indeed, it has been "argued that widespread testing could be counterproductive, in so far as this would divert scarce resources from prevention", and prevention "requires safe behaviors among people who are infected and among those who are or may be at risk" [23]. The second probability, $p$, represents the likelihood of a seropositive individual to transmit the disease to a non-seropositive sexual partner. This likelihood depends on the stage of the disease, thus individuals are also equipped with an age counter. The age counter $A_{i}(t)$ stands for the amount of time units that the individual $i$ has been in the system, at time $t$. An individual $i$ has a given sexual activity period $\tau_{i}$, after which he is replaced by a new individual. In other words, we have a constant population under deaths and births. The counter is also used to estimate in which stage of the disease an individual is at, and from 


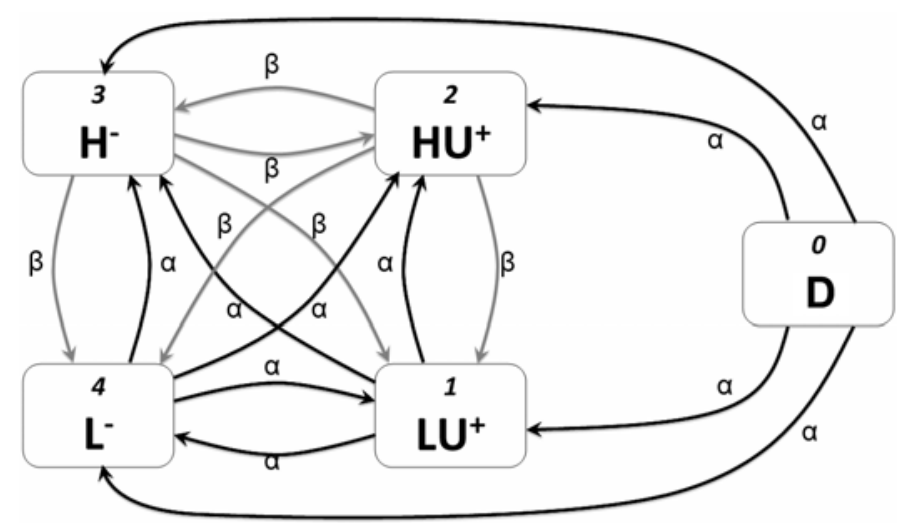

Unsafe social influence Safe social influence

Fig. 1. Social influences $v_{j i}$ are exerted upon an individual in class $i$ by an individual in class $j$. They are either encouraging a safe $(\alpha)$ or an unsafe $(\beta)$ behavior.

\section{TABLE I}

RULES FOR UPDATING A CELL $i$ AT TIME $t$ IN THE CELLULAR AUTOMATON

\begin{tabular}{|c|c|}
\hline State & Rules, in order of application \\
\hline $1-\mathrm{LU}^{+}$ & $\begin{array}{l}\text { a) If } C_{i}(t)<-1 \text { then start having a high-risk behavior } \\
\text { and become a } \mathrm{HU}^{+} \text {. } \\
\text { b) Opt for a very low-risk behavior } \mathrm{F} \text { with probability } \\
q \text {. }\end{array}$ \\
\hline $2-\mathrm{HU}^{+}$ & $\begin{array}{l}\text { a) If } C_{i}(t)>1 \text { then cease having a high-risk behavior } \\
\text { and become a } \mathrm{LU}^{+} \text {. } \\
\text { b) Opt for a very low-risk behavior } \mathrm{F} \text { with probability } \\
q \text {. }\end{array}$ \\
\hline $3-\mathrm{H}^{-}$ & $\begin{array}{l}\text { a) If } C_{i}(t)>1 \text { then cease having a high-risk behavior } \\
\text { and become a } \mathrm{L}^{-} \text {. } \\
\text { b) Become } \mathrm{HU}^{+} \text {with probability } p_{j} \text { for each partner } j \\
\text { in class } \mathrm{HU}^{+}, \mathrm{LU}^{+} \text {and } \mathrm{D} \text {. }\end{array}$ \\
\hline $4-\mathrm{L}^{-}$ & $\begin{array}{l}\text { a) If } C_{i}(t)<-1 \text { then start having a high-risk behavior } \\
\text { and become a H} \text {. } \\
\text { b) Become } \mathrm{HU}^{+} \text {with probability } p_{j} \text { for each partner } j \\
\text { in class } \mathrm{HU}^{+}, \mathrm{LU}^{+} \text {and } \mathrm{D} \text {. }\end{array}$ \\
\hline
\end{tabular}

there what is the likelihood of transmitting the disease. The rules governing an individual $i$ all start by replacing him after $\tau_{i}$ times, and are followed by two are summarized in Table I. When the epidemiological class of an individual changes after the rules, his social counter is reset to 0 .

\section{Simulations}

\section{A. Parameter Estimates}

In this section, we explain how the values of our parameters were estimated from the literature. We start by specifying in which proportions our population is divided into epidemiological classes. Then, given the class of an individual, we determine his number of sexual partners. Finally, an individual may be infected by HIV through a partner with a probability depending on the stage at which the partner is. Individuals eventually die, either when they reach the regular end of sexual period or earlier when living with HIV. They are then replaced by individuals with an initial sexual activity age set to 0 , and an epidemiological class chosen using the initial distribution of the population. All simulations, in the next two sections, use these parameters as well as a two dimensional toroidal cellular automaton of grid size $30 \times 30$, and were ran for 2000 times

Initially, we consider that there are no seropositive individuals who opted for a very low-risk behavior, since they are considered as an outcome of the system. Thus, the population consists of individuals in either a low or a high-risk group. The latter is often called the core group, and is known to be a minority. However, estimates of the core group vary tremendously since definitions swarm through different studies: for example, the core group in the overall population can be defined as five or more new partners per year [24] whereas this rate corresponds to an amount per day in populations at risks such as sex workers [25]. Our modelling choice is that an individual will be assigned to a high-risk group with a probability of $35 \%$, and thus to a low-risk group with the complementary probability $65 \%$. Given that each group is further divided according to sexual activities (as in [26]) and seropositivity, the probabilities are $20 \%, 10 \%, 25 \%$, and $45 \%$ for an individual to be in group $\mathrm{LU}^{+}, \mathrm{HU}^{+}, \mathrm{H}^{-}$, and $\mathrm{L}^{-}$ respectively.

The social network of an individual is a randomized neighborhood whose size depends on the epidemiological class of the individual. Indeed, by definition, individuals at lowrisk have less unprotected sexual encounters per week than individuals at high-risk. This difference in the number of partners has been advocated as an important feature to explain why some populations such as men having sex with men were more at risks than others [27]. However, estimates of the number of such encounters per week vary largely across populations: for example, the number of clients of sex workers can range from three to six per day [25], and the rate of requesting condom use as well as the chance of rejection can bear no resemblance between different settings. Thus, modelling choices are necessary. We abstracted the number of unprotected weekly sexual encounters in the low-risk category to be between 0 and 2, whereas it is 3 or 4 in the high-risk category. Each time step stands for a week thus individuals at low-risk are assigned between 0 and 2 neighbors randomly at each time step, and 3 or 4 for high-risk. Previous studies have shown that the prevalence of high-risk sexual behavior decreases when an individual is aware of being seropositive. A meta-analysis based on 11 articles found an average decrease of 53\% and the decrease ranged from $25 \%$ to $65 \%$ [28]. We incorporate this aspect by considering that people living with HIV and aware of it have around half as many sexual partners as other people. Thus, at a given time step, such individuals have at most one sexual partner, which translates to being assigned either zero or one neighbor.

The average probability of transmitting HIV from one individual to his sexual partner is called the HIV infectivity. An individual infected with HIV undergoes several stages (primary or acute, latency, AIDS) during which the viral concentration in blood and semen varies. The infectivity peaks in the acute stage and it has been suggested that infection at this stage may "account for a great part of the risk of transmis- 
sion" [29]. Thus, we denote by $p_{a}$ the infectivity in the acute stage, and $p_{r}$ the infectivity in the remaining stages (latency and AIDS). We assign $p_{a}$ to be the average of the maximum infectivity per-contact across types of sexual intercourse, and similarly $p_{r}$ as the average of the minimum. Using the values from [29], this leads to $p_{a}=0.05$ and $p_{r}=0.005$. As the authors argued, a probability such as 0.056 may correspond to the acute stage whereas 0.003 to 0.0014 may represent a later stage. More realistic estimates of the infectivity would require additional factors at the individual level (gene mutations, male circumcision, vaginal flora, other sexually transmitted infections, etc.) and between individuals (type of sexual act and direction of transmission) [30]. Such factors could be taken into account by introducing new epidemiological classes: for example, modelling a greater infectivity rate from male to female than from female to male (direction of transmission) could be performed by subdividing each class into male and female (e.g., $\mathrm{LU}^{+}$becomes $\mathrm{LU}_{m}^{+}$and $\mathrm{LU}_{f}^{+}$) [31]. While this would certainly benefit to the realism, it may not yield major changes for the overall dynamic of the system, which is the goal of the simulations.

A study on 13,030 individuals from 15 countries summarizes the age at seroconversion and the corresponding survival in years [32]. We obtain an indicator of survival using a weighted sum. For example, $31 \%$ of individuals were aged 15 to 24 at seroconversion and survived 12.5 years, thus they are expressed as the term $0.31 \times 12.5$ in the weighted sum. This method leads to an average survival of 10.82 years. Regarding the life expectancy of sexual activity, we use the estimate of 35 years from a report of the Center for HIV Identification, Prevention, and Treatment Services (CHIPTS) [33]. We assign the initial 'sexual age' of individuals to be between 0 and 35 using a uniform distribution.

\section{B. Simulation Results}

1) Impact of Promoting Safer Behaviors: With a probability $q=0.25 \%$ of testing per week and an influence $\beta=0.03$ promoting unsafe behaviors, we observed that a small influence $\alpha$ toward safe behaviors could have a major impact. If we assume a pessimistic estimate of $\alpha=0.005$ (i.e., the tendency to promote unsafe behaviors is six times greater), then an oscillatory configuration leads to $90 \%$ of individuals living with HIV. However, if we assume $\alpha=0.01$, which is still lower than $\beta$, the number of such individuals decreases to approximately $80 \%$. Both situations are illustrated in Figure 2, where thick curves show seropositive individuals and thin curves show non-seropositive individuals. One of the most noticeable changes between the two configurations is that we go from an outcome with a majority of individuals having a highrisk behavior to a situation in which they became a minority. This suggests that influences toward safe behavior can lead to significant improvements even with stronger tendencies toward unsafe behavior, assuming a small probability of testing.

2) Probability of HIV Testing: We studied the two settings of the previous section and doubled $q$ to $0.5 \%$. This achieved a reduction in the proportion of individuals living with HIV to 0.8 , mainly due to the increase of individuals in class $\mathbf{D}$ who changed their behavior after positive testing. Furthermore, as illustrated in Figure 3, the trend in the population is progressively reversed as the proportion of individuals with a high-risk sexual behaviour tends to zero. The improvement showed in Figure 3(a) over Figure 2(a) is similar to the change in $\alpha$ shown in Figure 2(b): both increase the proportion of ideal class of non-seropositive individuals with a low-risk sexual behavior from an almost null value to 0.2 . The advantage of improving both $\alpha$ and $q$, shown in Figure 3(b) is not significant at the end of the simulation but yields the same benefits earlier (at 600 weeks instead of 1600).

3) Overall Behavior: When we kept $\beta$ and $q$ fixed, and only varied $\alpha$, we observed that the system was bistable: there is a threshold for $\alpha$ below which there is a majority of seropositive individuals, and after which the majority of the population is non-seropositive with a low-risk behavior. Since the transition between the two states was obtained directly by varying $\alpha$, this is called a first-order phase transition and is common to numerous systems modelled in physics. The same behaviour was found when keeping $\alpha$ and $\beta$ fixed, and varying $q$. This suggests that attention should still be paid to policies that yield small improvements in $\alpha$ or $q$ for a given community, since it can reverse the trends in the population.

The particular values of $\alpha$ and $q$ at which the population turns to a beneficial stage strongly depend on the initial distribution in epidemiological classes. For example, in a population with a majority of individuals having a high-risk behavior $\left(10 \% \mathbf{L U}^{+}, 20 \% \mathbf{H U}^{+}, 45 \% \mathbf{H}^{-}, 25 \% \mathbf{L}^{-}\right)$and the same values $\beta=0.03, q=0.25 \%$, a much stronger $\alpha=0.03$ (versus 0.01 previously) is needed to achieve the state with a healthy majority.

\section{CONCLUSIONS}

We modelled the spread of HIV between individuals by taking into account numerous features shown to matter in the real world: type of sexual behavior, impact of testing, explicit sexual contact with different frequencies depending on the sexual behavior, infectivity depending on the stage of the disease, sexual activity period, and social influences. By focussing on the latter, we have shown that promoting a safe sexual behavior in a population could be in the same order of efficiency as HIV testing. If there is a minority of individuals with a high-risk sexual behavior, then the influence toward safe behavior can be lower than the one toward unsafe behavior and yet the overall health of the population can improve. However, as it may be found in high-risk populations, a higher quantity of individuals with a high-risk sexual behavior may require a much higher influence in the community to achieve the same benefits. This study could potentially be used in an optimization manner: by calculating the cost of HIV testing and the promotion of safe behavior, it is possible to search the most cost-effective measure. The search could also be performed by measuring other resources than the raw cost. A particularly interesting approach is to obtain measures for 

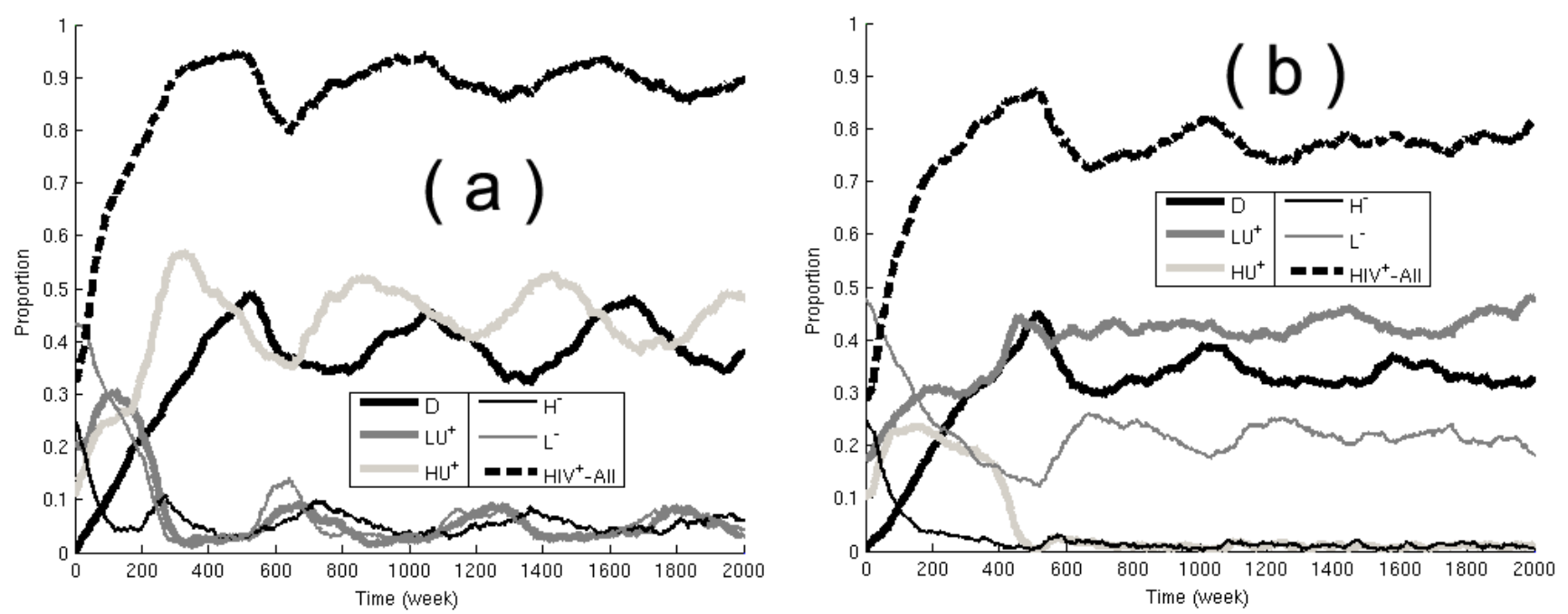

Fig. 2. Effect of the safe social influence $\alpha$ for $\beta=0.03, q=0.25 \%, \alpha=0.005$ (a) and $\alpha=0.01$ (b).
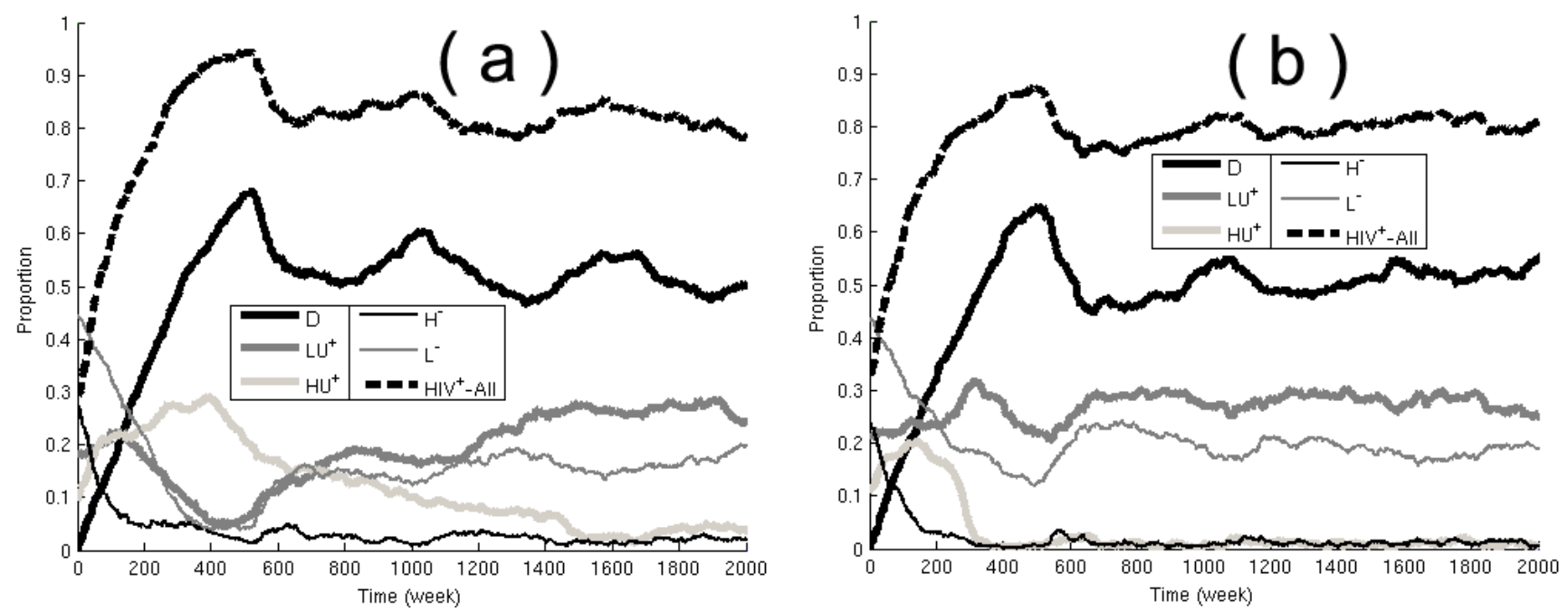

Fig. 3. Effect of the probability of testing $q=0.5 \%$ for $\beta=0.03, \alpha=0.005$ (a) and $\alpha=0.01$.

different populations (i.e., different types of proportions of individual following specific sexual behaviors).

We used the known compartmental approach to model the population at a high level, and explicitly represented the sexual contacts using a cellular automaton. While cellular automata have been previously used to model HIV, it is a novel approach to consider a randomized neighborhood. This accounts for sexual partners changing frequently, as is found in populations at risk. The topology (i.e., the contacts between partners) differs significantly from other cellular automata in which contacts are fixed. Further research could refine the rules of the automaton and introduce additional conditions on random neighborhood to better model target populations.

\section{ACKNOWLEDGMENTS}

The authors would like to thank Warren Hare, Kyle Vincent, and Philippe J. Giabbanelli for their input and helpful discussions. The authors are grateful to the support from the CTEF
Modelling of Complex Social Systems (MoCSSy) Program and the IRMACS Centre at Simon Fraser University while conducting the research presented in this paper. In addition, HHT would also like to acknowledge the support in part from the Natural Sciences and Engineering Research Council of Canada's (NSERC) Postdoctoral Fellowship.

\section{REFERENCES}

[1] UNAIDS, "Interactive map: Towards universal access," 2009.

[2] Y. A. Amirkhanian and J. A. Kelly, "A social network-level HIV intervention program in Central and Eastern Europe: accessing at-risk, hard-to-reach populations," no. E10655. The XV International AIDS Conference, 2004.

[3] K. A. Tyler and K. A. Johnson, "Trading sex: voluntary or coerced? the experiences of homeless youth," Journal of Sex Research, vol. 43, pp. 208-216, 2006.

[4] E. Rice, N. G. Milburn, and M. J. Rotheram-Borus, “ Pro-social and problematic social network influences on HIV/AIDS risk behaviours among newly homeless youth in Los Angeles," AIDS Care, vol. 19, pp. 697-704, 2007. 
[5] E. Rice, J. A. Stein, and N. Milburn, "Countervailing social network influences on problem behaviors among homeless youth," Journal of Adolescence, vol. 31, no. 5, pp. 625-639, 2008.

[6] L. Persson, P. O. Ostergren, B. S. Hanson, A. Lindgren, and A. Naucler, "Social network, social support and the rate of decline of CD4 lymphocytes in asymptomatic HIV-positive homosexual men," Scandinavian $j$. of public health, vol. 30, no. 3, pp. 184-190, 2002.

[7] K. Sullivan, "Male self-disclosure of hiv infection to sex partners: a hawaii-based sample," Journal of the association of nurses in AIDS care, vol. 20, no. 6, pp. 442-457, december 2009.

[8] L. A. Eaton, S. C. Kalichman, D. N. Cain, C. Cherry, H. L. Stearns, C. M. Amaral, J. A. Flanagan, and H. L. Pope, "Serosorting sexual partners and risk for hiv among men who have sex with men," Am. J. of Preventive Medicine, vol. 33, no. 6, pp. 479-485, 2007.

[9] H. W. Hetcote, "The mathematics of infectious diseases," SIAM Review, vol. 42, no. 4, pp. 599-653, 2000.

[10] A. S. Perelson and P. W. Nelson, "Mathematical analysis of hiv-1 dynamics in vivo," SIAM Review, vol. 41, no. 1, pp. 3-44, 1999.

[11] M. Kremer, "Integrating behavioral choice into epidemiological models of aids," Q. J. Econ., vol. 111, no. 2, pp. 549-573, 1996.

[12] S. Bansal, B. T. Grenfell, and L. A. Meyers, "When individual behaviour matters: homogeneous and network models in epidemiology," Journal of the Royal Society Interface, vol. 4, no. 16, pp. 879-891, 2007.

[13] A. Ilachinski, The Cellular Automata. A Discrete Universe. World Scientific Publishing Co., Inc., River Edge, New Jersey, 2001.

[14] S. Venkatachalam and A. Mikler, "Towards computational epidemiology: Using stochastic cellular automata in modeling spread of diseases," in Proc. of the $4^{\text {th }}$ Annual International Conference on Statistics, January 2005.

[15] R. Hegselmann and A. Flache, "Understanding complex social dynamics: A plea for cellular automata based modelling," in $J$. of Artificial Societies and Social Sim. 1(3), 1998.

[16] M. J. Fresnadillo, E. Garcia, J. E. Garcia, A. Martin, and G. Rodriguez, "A sis epidemiological model based on cellular automata on graphs," Lecture Notes in Computer Science, vol. 5518, pp. 1055-1062, 2009.

[17] P. Figueirdo, S. Coutinho, and R. Z. dos Santos, "Robustness of a cellular automata model for the HIV infection," Physica A, vol. 387, no. 26, pp. 6545-6552, 2008.

[18] A. Benyoussef, N. E. HafidAllah, A. ElKenz, H. Ez-Zahraouy, and M. Loulidi, "Dynamics of hiv infection on 2d cellular automata," Physica A, vol. 322, pp. 506-520, 2003.

[19] R. B. Schinazi, "On the importance of risky behavior in the transmission of sexually transmitted diseases," Mathematical Biosciences, vol. 173, pp. 25-33, 2001.

[20] J. M. Read, K. T. Eames, and J. W. Edmunds, "Dynamic social networks and the implications for the spread of infectious disease," Journal of the Royal Society Interface, vol. 5, no. 26, pp. 1001-1007, 2008.

[21] A. Tripathi, R. Naresh, and D. Sharma, "Modelling the effect of screening of unaware infectives on the spread of hiv infection," Appl. Math. \& Comp., vol. 184, pp. 1053-1068, 2007.

[22] N. C. Grassly, C. M. Lowndes, T. Rhodes, A. Judd, A. Renton, and G. P. Garnett, "Modelling emerging hiv epidemics: the role of injecting drug use and previous sexual transmission in the russian federation, china and india," Int. J. of Drug Policy, vol. 14, no. 1, pp. 25-43, 2003.

[23] R. Danziger, "The social impact of hiv testing: a comparative analysis of britain and sweden," Social Science and Medicine, vol. 48, pp. 293-300, 1999.

[24] H. Stigum, W. Falck, and P. Magnus, "The core group revisited: the effect of partner mixing and migration on the spread of gonorrhea, chlamydia and hiv," Mathematical Biosciences, vol. 120, pp. 1-23, 1994

[25] G. Ohi, I. Uemuro, A. Akabayashi, I. Kai, M. Miyasaka, and K. Naka "Preventive behaviors against hiv transmission adopted by japanese commercial sex workers (csws)," Environmental health and preventive medicine, vol. 3, pp. 132-134, 1997.

[26] C. Sumodhee, J. Hsieh, C. Sun, C. Huang, and A. Y. Chen, "Impact of Social Behaviors on HIV Epidemic: A Computer Simulation View," Int. Conf. on Computational Intelligence for Modelling, Control and Automation, vol. 2, pp. 550-556, 2005.

[27] G. Rotello, Sexual Ecology: AIDS the destiny of gey men. Dutton, 1997.

[28] G. Marks, N. Crepaz, J. W. Senterfitt, and R. S. Janssen, "Meta-analysis of high-risk sexual behavior in persons aware and unaware they are infected with hiv in the united states," J. of Acquired Immune Defic. Syndr., vol. 39, no. 4, pp. 446-453, 2005.
[29] R. A. Royce, A. Sena, W. C. Jr., and M. S. Cohen, "Sexual transmission of HIV," The New England Journal of Medicine, vol. 336, pp. 10721078, 1997.

[30] K. A. Powers, C. Poole, A. E. Pettifor, and M. S. Cohen, "Rethinking the heterosexual infectivity of hiv-1: a systematic review and meta-analysis," Lancet Infect. Dis., vol. 8, pp. 553-563, 2008.

[31] Z. Mukandavire, C. Chiyaka, W. Garira, and G. Musuka, "Mathematical analysis of a sex-structured hiv/aids model with a discrete time delay," Nonlinear analysis, vol. 71, pp. 1082-1093, 2009.

[32] Collaborative Group on AIDS Incubation and HIV Survival Including the CASCADE EU Concerted Action, "Time from HIV-1 seroconversion to AIDS and death before widespread use of highly-active antiretroviral therapy: a collaborative re-analysis," The Lancet, vol. 355, pp. 11311137, 2000.

[33] Center for HIV Identification, Prevention, and Treatment Services (CHIPTS), "Anticipating the efficacy of hiv preexposure prophylaxis (prep) and the needs of at-risk californians," in AIDS Partnership California, 2004. 